\title{
Microelectromechanical Systems-Based Neurotools for Non-Invasive Ultrasound Brain Stimulation
}

\author{
Yehhyun Jo, Chaerin Oh, and Hyunjoo Jenny Lee \\ School of Electrical Engineering, Korea Advanced Institute of Science and Technology, Daejeon, Korea
}

\begin{abstract}
In the current aging society, the number of patients suffering from degenerative brain diseases is continuously increasing. However, many of these brain disorders are intractable and difficult to treat. Non-invasive brain stimulation is an attractive alternative method to a pharmaceutical approach that attempts to treat brain disorders through physical stimulation. Among the various direct brain stimulation techniques, such as electrical, magnetic, and optical, ultrasound has been proposed as a new modality for neuromodulation due to its distinct advantages such as high spatial resolution and in-depth targeting. As ultrasound modality is still in the early stages of development, further investigations on various aspects such as neuromodulation mechanism, therapeutic effects, and safety are still required. Although ultrasound technology is a mature biomedical tool developed from ultrasound imaging, many new technological advancements such as miniaturized devices based on microelectromechanical systems technology have been recently introduced for the specific purpose of neuromodulation. In this review, we introduce these new neurotools which are essential to uncovering the fundamental mechanisms of ultrasound brain stimulation and ultimately to developing an effective therapeutic means for brain disorders.
\end{abstract}

Key Words: Ultrasound neuromodulation; Non-invasive brain stimulation; MEMS; Capacitive micromachined ultrasound transducers

Received: May 27, 2019 Revised: June 10, 2019 Accepted: June 11, 2019

Corresponding author: Hyunjoo Jenny Lee, PhD, School of Electrical Engineering, Korea Advanced Institute of Science and Technology, 291 Daehak-ro, Yuseong-gu, Daejeon 34141, Korea.

Tel: 82-42-350-7436, Fax: 82-42-350-7636, E-mail: hyunjoo.lee@kaist.ac.kr

(a) This is an Open Access article distributed under the terms of the Creative Commons Attribution Non-Commercial License (https://creativecommons.org/licenses/bync/4.0) which permits unrestricted non-commercial use, distribution, and reproduction in any medium, provided the original work is properly cited.

\section{INTRODUCTION}

While the number of people suffering from brain disorders continues to increase in the modern aging society, it is still a challenge to develop effective treatments for many of these diseases. For example, the risk of Alzheimer's disease (AD), an age-dependent disorder, doubles every five years after the age of 65 . However, there is still no medication to treat $\mathrm{AD}$. While pharmaceutical means offers the highest patient compliance, it is difficult to discover an effective drug for brain disorders because of the existence of the brain-blood barrier (BBB) within the brain capillary endothelia [1]. BBB, a tight junction that exists between the blood vessel and extracellular space of the brain, allows passage of only a fraction of small-molecule drugs. In addition to this physical limitation, the pharmacological approach suffers from drug resistance, sideeffects, tolerance, and dependence, which further complicates the pharmaceutical method to treat brain disorders.

Thus, direct brain stimulation serves as a promising alternative for the treatment of brain disorders. A number of modalities, such as electrical, magnetic, and optical, have been explored to treat brain disorders and modulate brain activities. Electrical stimulation, such as transcranial direct current stimulation (tDC), transcranial alternating current stimulation (tAC), and deep brain stimulation (DBS), is the most commonly used method of direct brain stimulation [2-4]. Although invasive, DBS is currently clinically used to treat Parkinson's disease through implantation of a long probe to the target region of the brain [3,5]. A relatively new modality, optical stimulation, is based on genetic modification of cells with light-sensitive opsins and thus allows for cell-type specific stimulation with high spatial resolution [6]. However, while DBS and optical stimulation are capable of achieving high spatial resolution and deep penetration depth, the operation is highly in- 
vasive, which results in low patient compliance and limited clinical application. Transcranial magnetic stimulation (TMS), which induces electrical activity within the brain through a magnetic field, is widely used for the treatment of depression [7]. However, TMS is limited to the stimulation of shallow regions and it is difficult to achieve high spatial resolution [8].

Deep brain regions such as the subthalamic nucleus, globus pallidus, and ventral intermediate nucleus of the thalamus are often associated with many critical brain circuits and stimulation of such regions have shown to alleviate the symptoms of various disorders, such as pain, tremor, depression, and addiction [9-11]. Yet, there have been no reports of a non-invasive stimulation modality that has targeted these deep brain regions with high spatial resolution. Non-invasiveness is a crucial feature of neuromodulation as high patient compliance implies an increase in the number of applicable pathologies. Among the various direct brain stimulation modalities, optogenetics is highly invasive, TMS is limited in penetration depth, and electrical stimulation suffers from low spatial resolution. Thus, there is an imperative need for a non-invasive modality, which is capable of both in-depth targeting and high spatial resolution.

Low-intensity transcranial focused ultrasound stimulation (LIFU or tFUS) is a promising modality for direct brain stimulation because of its inherent advantage of focusing capability [12]. Ultrasound can be focused either through a mechanical means using an acoustic lens or through an electrical means using a beam-forming array of ultrasound transducers. In addition to beam-steering capabilities, tFUS offers various advantages such as non-invasiveness, in-depth targeting, and technical maturity of ultrasound technology [13]. By calibrating for attenuation and scattering through the skull, an ultrasound beam with a focal point away from the transducer surface can target deep brain regions without exposing the other regions within its focal width to a strong intensity of ultrasound. Most importantly, because of the long history of ultrasound medical imaging, technological and biophysical aspects such as beam-forming, circuit integration, magnetic resonance (MR) compatibility, skull and scattering modeling, transducer design, and biological effects are well established [14-16].

Consequently, many studies have been conducted on tFUS over the past decade including investigations into in vivo effects in small animals to large non-human primates [17-25], neuromodulation mechanisms [26-28], safety [12], and stimulation protocols [28]. Compared to other direct brain stimulation modalities, ultrasound stimulation is a relatively new methodology and requires further investigation of the exact neuromodulation mechanisms, therapeutic effects, and safety as a potential clinical tool. However, in order to investigate in vivo and in vitro effects, the current tFUS device technology is limited in terms of size, portability, and spatial resolution. For example, due to the abundance of rodent disease models, mice in vivo experiments are useful in the evaluation of the therapeutic effects of tFUS. However, ultrasound transducer systems used for mice in vivo experiments are not only heavy and bulky but also require large collimators [20-22]. Thus, there is a need to develop a new set of neurotools based on microelectromechanical systems (MEMS), which enables fabrication of devices at the micron scale with high accuracy and reproducibility. In this review, the new types of MEMS-based neurotools specifically developed for in vitro and in vivo tFUS is summarized in detail.

\section{IN VITRO NEUROTOOLS}

The neuromodulation mechanism of ultrasound must be clearly understood in order for tFUS to be accepted as a new treatment in clinical applications. Although many hypotheses have been proposed, there is yet a universal agreement on the exact mechanism of ultrasound neuromodulation $[13,26,27,29,30]$. Hypotheses on heat activation and modulation of membranes due to cavitation have been proposed but are limited to higher intensities of ultrasound stimulation than that required for neuromodulation [30]. New hypotheses focus on the biophysical effects of acoustic radiation force generated by ultrasound such as modulation of mechanosensitive channels and mechanical plasma deformation $[27,29,30]$.

For evaluating the effects of radiation force in the cellular level, the existing ultrasound stimulation technology is too bulky to devise a compact system and it is difficult to devise a single system with variable frequency, variable intensity, and high throughput. For example, the work on ultrasound neuromodulation of sciatic nerves used a commercially available bulky transducer with a 1.1 $\mathrm{MHz}$ center frequency [31]. Not only was the focal area large with an ellipsoidal focal region ( $1.3 \mathrm{~mm}$ wide and $9.7 \mathrm{~mm}$ long), but the overall system was also tens of centimeters in size because the width of the transducer was $6.5 \mathrm{~cm}$ (Figure 1A). On the other hand, MEMS-based ultrasound transducers, such as capacitive micromachined ultrasound transducers (CMUT), piezoelectric micromachined ultrasound transducers (PMUT), and piezoelectric ultrasound transducers (PUT), allow for a wide range of experiments to investigate ultrasound neuromodulation mechanisms. By utilizing micromachining technology, ultrasound transducers with a wide range of frequencies, varying intensities, and diverse shapes (e.g., linear, circular, and ring arrays) can be readily fabricated [32-34].

For in vitro application, Ko et al. [35] demonstrated for the first time a PMUT array capable of neuromodulation of cultured cells with a high spatial resolution. The proposed PMUT array consisted of 16 elements [one-dimensional (1D) array] and exhibited a resonant frequency of $780 \mathrm{kHz}$ in immersion with $8 \mathrm{~mW} / \mathrm{cm}^{2}$ of spatial-peak temporal-average intensities $\left(\mathrm{I}_{\text {SPTA }}\right)$ (Figure 1B). The fabricated device was custom-packaged on a printed circuit board (PCB) with a biocompatible polydimethylsiloxane (PDMS) chamber. This setup allowed for continuous flow of medium to sustain the viability of the cultured cells. To observe the ultrasound neuromodulation using the PMUT device, transient receptor potential ankyrin 1 (TRPA1) channels, a mechanoreceptor that mediates intracellular $\mathrm{Ca}^{2+}$ transient, were expressed in the human embryonic kidney (HEK) 293T cells. By using Fura-2 ratiometric 

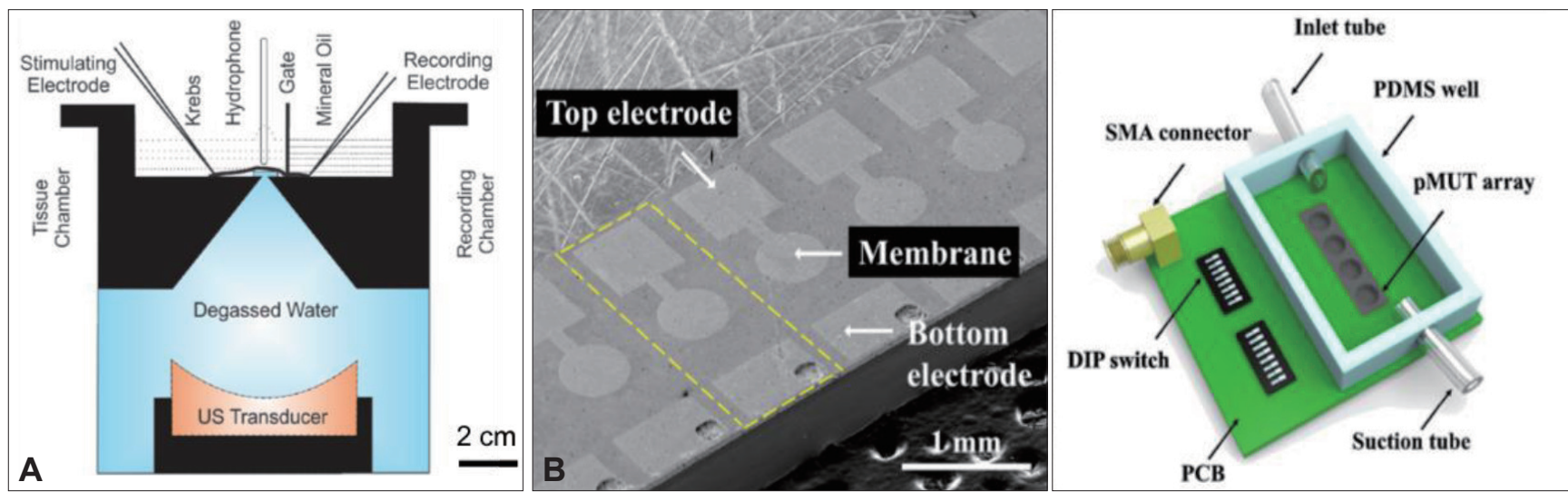

Figure 1. In vitro systems for ultrasound stimulation. A: Schematic diagram of the in vitro system utilizing a commercially available transducer; Reprinted from llham. Ultrasonic neuromodulation in vitro at single neuron resolution 2017, with permission of University of Connecticut [31]. B: Scanning electron microscope image of the PMUT array (left) and schematic diagram of the compact overall system (right); Reprinted from Ko et al. 28th IEEE Int Conf Micro Electro Mechan Syst (MEMS) 2015:651-654, with permission of IEEE [35]. PMUT: piezoelectric micromachined ultrasound transducers, PDMS: polydimethylsiloxane, SMA: SubMiniature version A, DIP: dual in-line package, PCB: printed circuit board.
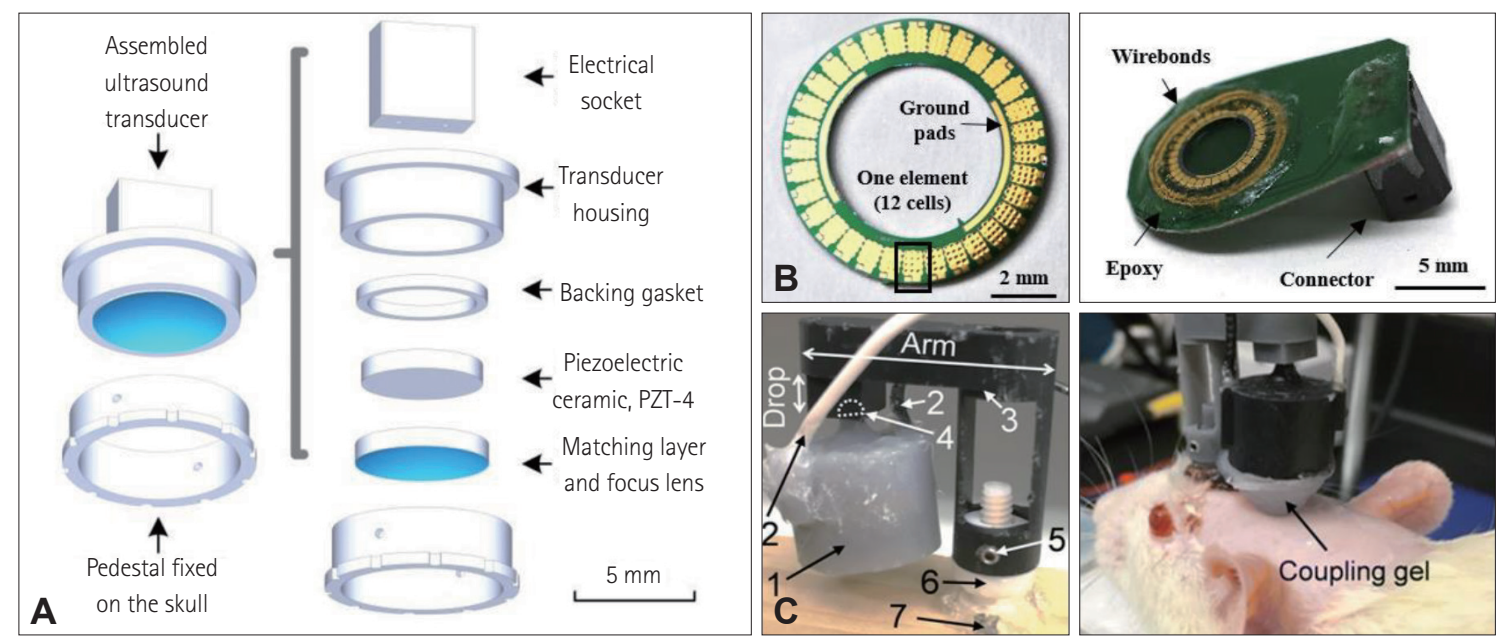

Figure 2. Schematics and descriptions of three different miniature ultrasound transducers. A: Assembly of miniaturized ultrasound transducer; Reprinted from Li et al. IEEE Trans Biomed Eng 2019;66:217-224, with permission of IEEE [40]. B: Images of capacitive micromachined ultrasound transducers ring array (left) and fully packaged device (right); Reprinted from Kim et al. Brain Stimul 2009;12:251255, with permission of Elsevier [39]. C: Images of wearable miniature ultrasound transducer on wooden board (left) and mounted on an anesthetized rat (right); Reprinted from Lee et al. BMC Neurosci 2018;19:57, with permission of Springer [41].

Table 1. Characteristics of miniaturized transducers

\begin{tabular}{lcccccc}
\multicolumn{1}{c}{ Study } & $\begin{array}{c}\text { Device diameter } \\
(\mathrm{mm})\end{array}$ & $\begin{array}{c}\text { Resonant frequency } \\
(\mathrm{kHz})\end{array}$ & $\begin{array}{c}\text { Focal length } \\
(\mathrm{mm})\end{array}$ & $\begin{array}{c}\text { Spatial resolution (lateral) } \\
(\mathrm{mm})\end{array}$ & $\begin{array}{c}\text { Fabrication } \\
\text { method }\end{array}$ & $\begin{array}{c}\text { Freely-moving } \\
\text { system }\end{array}$ \\
Li et al. [40] & 5.0 & 2000 & 7.0 & 1.20 & Piezoelectric ceramic & Possible \\
Kim et al. [39] & 8.1 & 183 & 2.3 & 2.75 & MEMS & $\begin{array}{c}\text { Possible } \\
\text { Lee et al. [41] }\end{array}$ \\
\hline
\end{tabular}

MEMS: microelectromechanical systems.

$\mathrm{Ca}^{2+}$ imaging, successful neuromodulation using the PMUT was demonstrated for the first time. Compared to the 6.4-cm-wide single-element commercial transducer, the newly developed 1Darray PMUT fabricated using MEMS technology consisted of $500-\mu \mathrm{m}$-diameter elements, which allows for highly localized targeting. Thus, further development of MEMS-based ultrasound neurotools could allow simultaneous neuromodulation of different ultrasound frequencies and intensities.

\section{IN VIVO NEUROTOOLS}

MEMS-based in vivo neurotools are also essential in expanding the horizon of ultrasound neuromodulation. These tools not only facilitate in vivo experiments to uncover the mechanism of neuromodulation, but also allows neuroscientists to evaluate the effects of ultrasound on various brain functions. While the investigation into neuromodulation mechanisms is often conducted in vitro, recently, two in vivo experiments have been reported 
that demonstrated an indirect pathway of ultrasound neuromodulation through auditory or cochlear neuronal circuits [36,37]. In addition, ultrasound modality, which is non-invasive and highly patient compliant, has great potential to be used to treat brain disorders. Thus, the effects of ultrasound neuromodulation on various brain functions, such as sensory, motor, and cognition, need to be investigated. Since the effects are often monitored through behavior studies, ultrasound neuromodulation in vivo setup must be compact and light to accommodate freely-moving animal experiments. In addition, when such freely-moving systems are developed, it would be possible to observe the long-term safety of ultrasound neuromodulation without the need for anesthesia and stereotaxic fixation.

Since MEMS technology enables fabrication of miniaturized devices with high accuracy and versatile functionality, MEMSbased ultrasound transducers are excellent candidates for behavioral studies. Most of the previous works demonstrated ultrasound neuromodulation on immobile or sedated animals because commercially available transducers are often bulky, heavy, and requires a collimator [19-25]. Recently, three pioneering works on MEMS-based ultrasound transducers have successfully demonstrated in vivo neuromodulation in freely-moving behaving animals [38-41]. The first of these reports was based on a CMUT ring array composed of 32 elements with a $183-\mathrm{kHz}$ resonant frequency $[38,39]$. The overall system was $0.73 \mathrm{~g}$ in weight and no collimator was required for the system (Figure 2).

The other two works were based on piezoelectric transducers (Table 1) [40,41]. Lee et al. [41] developed a miniaturized tFUS system for freely-moving animals. The transducer was about $6 \mathrm{~g}$ in weight and packaged in a three-dimensional-printed wearable headgear. The developed freely-moving system was verified by performing tFUS in awake and anesthetized groups. Li et al. [40] optimized the size of the PZT and produced piezoelectric transducers with $5 \mathrm{~mm}$ in diameter and $0.49 \mathrm{~g}$ in weight. By attaching a concave epoxy acoustic lens in front of the transducer, a lateral spatial resolution of $1.2 \mathrm{~mm}$ was achieved. The effect of ultrasonic neuromodulation was demonstrated by observing the changes in the in situ electrophysiological signals. In addition to these works, beam-forming circuitry has been implemented with the CMUT devices for the application of in vivo ultrasound neuromodulation [42]. These systems offer a greater level of versatility to the current neuromodulation in vivo experiments by allowing behaviors studies on various characteristics such as cognition, social behavior, and sleep.

\section{CONCLUSION}

With the convergence of ultrasound technology and neuroscience, new neurotools are actively being developed for more versatile in vitro and in vivo experiments. MEMS-based devices such as PMUTs and CMUTs are excellent candidates for investigating the mechanisms, effects, and safety of ultrasound neuromodulation because of the light and compact structure and multifunctional capabilities. Since these developments are relatively new, we expect further improvements and advancement with regards to MR compatibility, operational reliability, biological safety, and translatability to clinical applications.

\section{Acknowledgments}

This research was supported by the Brain Research Program through the National Research Foundation of Korea (NRF) funded by the Ministry of Science and ICT (No. 2016M3C7A1904343) and by the National Research Foundation of Korea (NRF) grant funded by the Korea government (MSIT) (No. 2019R1A2C2002084).

\section{Conflicts of Interest}

The authors have no potential conflicts of interest to disclose.

\section{Author Contributions}

Conceptualization: Hyunjoo Jenny Lee. Data curation: Yehhyun Jo. Formal analysis: Chaerin Oh. Funding acquisition: Hyunjoo Jenny Lee. Investigation: Chaerin Oh and Yehhyun Jo. Methodology: Yehhyun Jo. Project administration: Hyunjoo Jenny Lee. Supervision: Hyunjoo Jenny Lee. Validation: Chaerin Oh. Writing-original draft: Yehhyun Jo and Chaerin Oh. Writing-review \& editing: Hyunjoo Jenny Lee.

\section{ORCID iDs}

Hyunjoo Jenny Lee (D)

https://orcid.org/0000-0001-9963-2515

Yehhyun Jo (D)

https://orcid.org/0000-0003-4731-240X

\section{REFERENCES}

1. Pardridge WM. The blood-brain barrier: bottleneck in brain drug development. NeuroRx 2005;2:3-14

2. Fregni F, Boggio PS, Nitsche MA, Marcolin MA, Rigonatti SP, Pascual-Leone A. Treatment of major depression with transcranial direct current stimulation. Bipolar Disord 2006;8:203-204

3. Deuschl G, Schade-Brittinger C, Krack P, Volkmann J, Schäfer H, Bötzel K, et al. A randomized trial of deep-brain stimulation for Parkinson's disease. $\mathrm{N}$ Engl J Med 2006;355:896-908.

4. Brunoni AR, Nitsche MA, Bolognini N, Bikson M, Wagner T, Merabet L, et al. Clinical research with transcranial direct current stimulation (tDCS): challenges and future directions. Brain Stimul 2012;5:175-195.

5. Rezai AR, Kopell BH, Gross RE, Vitek JL, Sharan AD, Limousin P, et al. Deep brain stimulation for Parkinson's disease: surgical issues. Mov Disord 2006; 21 Suppl 14:S197-S218.

6. Deisseroth K. Optogenetics. Nat Methods 2011;8:26-29.

7. George MS, Nahas Z, Molloy M, Speer AM, Oliver NC, Li XB, et al. A controlled trial of daily left prefrontal cortex TMS for treating depression. Biol Psychiatry 2000;48:962-970.

8. Opitz A, Legon W, Rowlands A, Bickel WK, Paulus W, Tyler WJ. Physiological observations validate finite element models for estimating subject-specific electric field distributions induced by transcranial magnetic stimulation of the human motor cortex. Neuroimage 2013;81:253-264.

9. Perlmutter JS, Mink JW. Deep brain stimulation. Annu Rev Neurosci 2006;29: 229-257.

10. Luigjes J, van den Brink W, Feenstra M, van den Munckhof P, Schuurman PR, Schippers R, et al. Deep brain stimulation in addiction: a review of potential brain targets. Mol Psychiatry 2012;17:572-583.

11. Johansen-Berg H, Gutman DA, Behrens TE, Matthews PM, Rushworth MF, 
Katz E, et al. Anatomical connectivity of the subgenual cingulate region targeted with deep brain stimulation for treatment-resistant depression. Cereb Cortex 2008;18:1374-1383.

12. Legon W, Sato TF, Opitz A, Mueller J, Barbour A, Williams A, et al. Transcranial focused ultrasound modulates the activity of primary somatosensory cortex in humans. Nat Neurosci 2014;17:322-329.

13. Tyler WJ. Noninvasive neuromodulation with ultrasound? A continuum mechanics hypothesis. Neuroscientist 2011;17:25-36.

14. Hynynen K, Clement GT, McDannold N, Vykhodtseva N, King R, White $\mathrm{PJ}$, et al. 500-element ultrasound phased array system for noninvasive focal surgery of the brain: a preliminary rabbit study with ex vivo human skulls. Magn Reson Med 2004;52:100-107.

15. Wygant IO, Jamal NS, Lee HJ, Nikoozadeh A, Oralkan O, Karaman M, et al. An integrated circuit with transmit beamforming flip-chip bonded to a 2-D CMUT array for 3-D ultrasound imaging. IEEE Trans Ultrason Ferroelectr Freq Control 2009;56:2145-2156.

16. Baker ML, Dalrymple GV. Biological effects of diagnostic ultrasound: a review. Radiology 1978;126:479-483.

17. Li GF, Zhao HX, Zhou H, Yan F, Wang JY, Xu CX, et al. Improved anatomical specificity of non-invasive neuro-stimulation by high frequency $(5 \mathrm{MHz})$ ultrasound. Sci Rep 2016;6:24738.

18. Fouragnan EF, Chau BKH, Folloni D, Kolling N, Verhagen L, Klein-Flügge $\mathrm{M}$, et al. The macaque anterior cingulate cortex translates counterfactual choice value into actual behavioral change. Nat Neurosci 2019;22:797-808.

19. Deffieux T, Younan Y, Wattiez N, Tanter M, Pouget P, Aubry JF. Low-intensity focused ultrasound modulates monkey visuomotor behavior. Curr Biol 2013;23:2430-2433.

20. Tufail Y, Matyushov A, Baldwin N, Tauchmann ML, Georges J, Yoshihiro A, et al. Transcranial pulsed ultrasound stimulates intact brain circuits. Neuron 2010;66:681-694.

21. Tufail Y, Yoshihiro A, Pati S, Li MM, Tyler WJ. Ultrasonic neuromodulation by brain stimulation with transcranial ultrasound. Nat Protoc 2011;6:14531470.

22. King RL, Brown JR, Pauly KB. Localization of ultrasound-induced in vivo neurostimulation in the mouse model. Ultrasound Med Biol 2014;40:15121522.

23. Downs ME, Buch A, Karakatsani ME, Konofagou EE, Ferrera VP. Bloodbrain barrier opening in behaving non-human primates via focused ultrasound with systemically administered microbubbles. Sci Rep 2015;5:15076.

24. Yoo SS, Bystritsky A, Lee JH, Zhang Y, Fischer K, Min BK, et al. Focused ultrasound modulates region-specific brain activity. Neuroimage 2011;56:12671275.

25. Kim H, Taghados SJ, Fischer K, Maeng LS, Park S, Yoo SS. Noninvasive transcranial stimulation of rat abducens nerve by focused ultrasound. Ultrasound Med Biol 2012;38:1568-1575.

26. Fomenko A, Neudorfer C, Dallapiazza RF, Kalia SK, Lozano AM. Low-intensity ultrasound neuromodulation: an overview of mechanisms and emerging human applications. Brain Stimul 2018;11:1209-1217.
27. Kubanek J, Shi J, Marsh J, Chen D, Deng C, Cui J. Ultrasound modulates ion channel currents. Sci Rep 2016;6:24170.

28. King RL, Brown JR, Newsome WT, Pauly KB. Effective parameters for ultrasound-induced in vivo neurostimulation. Ultrasound Med Biol 2013;39:312331.

29. Brohawn SG, Su Z, MacKinnon R. Mechanosensitivity is mediated directly by the lipid membrane in TRAAK and TREK1 K+ channels. Proc Natl Acad Sci U S A 2014;111:3614-3619.

30. Sassaroli E, Vykhodtseva N. Acoustic neuromodulation from a basic science prospective. J Ther Ultrasound 2016;4:17.

31. Ilham S. Ultrasonic neuromodulation in vitro at single neuron resolution [Master's Theses]. Storrs (CT): University of Connecticut, 2017. Available at: https://opencommons.uconn.edu/gs_theses/1173/.

32. Ladabaum I, Jin X, Soh HT, Atalar A, Khuri-Yakub BT. Surface micromachined capacitive ultrasonic transducers. IEEE Trans Ultrason Ferroelectr Freq Control 1998;45:678-690.

33. Nikoozadeh A, Oralkan Ö, Gencel M, Choe JW, Stephens DN, De La Rama A, et al. Forward-looking volumetric intracardiac imaging using a fully integrated CMUT ring array. IEEE Int Ultrason Symp; 2009 Sept 20-23; Rome, Italy. IEEE; c2009. p.511-514.

34. Park S, Yoon I, Lee S, Kim H, Seo JW, Chung Y, et al. CMUT-based resonant gas sensor array for VOC detection with low operating voltage. Sensor Actuat B Chem 2018;273:1556-1563.

35. Ko K, Lee J-H, Lee HJ, Oh S-J, Chun YE, Kim TS, et al. Micromachined ultrasound transducer array for cell stimulation with high spatial resolution. 28th IEEE Int Conf Micro Electro Mechan Syst (MEMS); 2015 Jan 18-22; Estoril, Portugal. IEEE; c2015. p.651-654.

36. Guo H, Hamilton M 2nd, Offutt SJ, Gloeckner CD, Li T, Kim Y, et al. Ultrasound produces extensive brain activation via a cochlear pathway. Neuron 2018;98:1020-1030.

37. Sato T, Shapiro MG, Tsao DY. Ultrasonic neuromodulation causes widespread cortical activation via an indirect auditory mechanism. Neuron 2018; 98:1031-1041.

38. Kim H, Kim S, Lee HJ. Capacitive Micromachined Ultrasonic Transducer (CMUT) ring array for transcranial ultrasound neuromodulation. Conf Proc IEEE Eng Med Biol Soc 2018;2018:2675-2678.

39. Kim H, Kim S, Sim NS, Pasquinelli C, Thielscher A, Lee JH, et al. Miniature ultrasound ring array transducers for transcranial ultrasound neuromodulation of freely-moving small animals. Brain Stimul 2009;12:251-255.

40. Li G, Qiu W, Zhang Z, Jiang Q, Su M, Cai R, et al. Noninvasive ultrasonic neuromodulation in freely moving mice. IEEE Trans Biomed Eng 2019;66: 217-224.

41. Lee W, Croce P, Margolin RW, Cammalleri A, Yoon K, Yoo SS. Transcranial focused ultrasound stimulation of motor cortical areas in freely-moving awake rats. BMC Neurosci 2018;19:57.

42. Seok C, Ali Z, Yamaner FY, Sahin M, Oralkan O. Towards an untethered ultrasound beamforming system for brain stimulation in behaving animals. Conf Proc IEEE Eng Med Biol Soc 2018;2018:1596-1599. 